\title{
Vortex Lattice of a Bose-Einstein Condensate as a Photonic Band Gap Material ${ }^{1}$
}

\author{
M. E. Taşgın ${ }^{a}$, Ö. E. Müstecaplığlu ${ }^{b}$, and M. Ö. Oktel ${ }^{a}$ \\ ${ }^{a}$ Department of Physics, Bilkent University, Bilkent, Ankara, 06800 Turkey \\ ${ }^{b}$ Department of Physics, Koç University, Sartyer, Istanbul, 34450 Turkey \\ e-mail: omustecap@ku.edu.tr \\ Received November 21, 2008
}

\begin{abstract}
Photonic crystal behavior of a rotating Bose-Einstein condensate with a triangular vortex lattice is reviewed and a scheme for getting much wider band gaps is proposed. It is shown that photonic band gaps can be widened an order of magnitude more by using a Raman scheme of index enhancement, in comparison to previously considered upper level microwave scheme.
\end{abstract}

PACS numbers: 03.75.Lm, 42.50.Gy, 42.70.Qs, 74.25.Qt

DOI: $10.1134 / \mathrm{S} 1054660 X 09040197$

\section{INTRODUCTION}

When a Bose-Einstein condensate (BEC) is rotated, after a certain critical frequency, vortices start to form. For high enough rotation frequency, these vortices become large in number and distribute in a periodical structure [1-4]. The usual structure is a triangular lattice, but other lattice types may as well occur, depending on the strength of the inter-particle interactions [5]. The density profile is periodically distributed vortices on an envelope function, which decreases toward the edge of the condensate $[3,4]$. The envelope is a slowly varying function compared to the vortex periodicity, such that few hundred vortices are observable experimentally [6]. The imaging of the lattice was accomplished while the condensate is in the trap in [5], different from the usual ballistic expansion imaging [1, 2, 79].

This periodicity simulates $2 \mathrm{D}$ photonic band gap (PBG) materials. Despite the common PBG material [10], however, the lattice parameter of a BEC vortex lattice is continuously tunable via the rotation frequency. Moreover, lattice type can be changed, while the BEC is in the trap, by controlling the inter-particle interaction strength by Feshbach resonances [11].

We recently proposed $[12,13]$ to directly measure the rotation frequency of $\mathrm{BEC}$ using the reflection from a directional PBG pulse propagation, stopped only in certain directions and allowed in others. The chopping in the reflected pulse, propagating through BEC lattice, measures its rotation frequency.

The difficulty is, however, to establish the high enough index contrast between the vortex cores and the bulk of the BEC. BEC is dilute gaseous atomic medium, as such exhibits dispersion and polarization only in the high absorptive frequency regimes. Neces-

\footnotetext{
${ }^{1}$ The article is published in the original.
}

sary contrast is achieved using one of the indexenhancement schemes $[14,15]$, which are based on the atomic quantum coherence. Coupling the excited (or ground) state to other auxiliary levels, it is possible to obtain high polarization response without absorption [15]. Quantum interference of various absorption paths allows the retain of the atoms in the excited level with cancelling absorption.

Dielectric response, obtained using index enhancement schemes, is complex in structure. Gain and absorption regimes crossover the zero absorption frequency and more over they are very strong (see Fig. 2). Dielectric function varies rapidly with the frequency and both real and imaginary parts of it changes sign. Checking the existence of the PBG at exactly on the zero-absorption frequency is straightforward. However, the determination of the frequency width of the band gap about such a point, surrounded by both gain and absorption, is sophisticated [13].

In this paper, we examine the photonic band structure of a triangular vortex lattice. We investigate the frequency width of the photonic band gap, for a complex frequency-dependent dielectric function. The unusual dielectric response is due to the index enhancement scheme [14], which is utilized for high index contrast. In order to be able to determine the band gap width, we also examine the complex Poynting vector beside the complex wave vector. We first review our previous results $[12,13]$ based upon upper level microwave scheme for index enhancement. After that we shall consider Raman scheme and show that it is capable to generate much wider band gaps.

Although in the literature there exists abundant of studies on photonic crystals (PC), the ones considering the complex frequency-dependent dielectric function are rare. There exist few studies, but these are focused on the examination of the effects of metallic compo- 


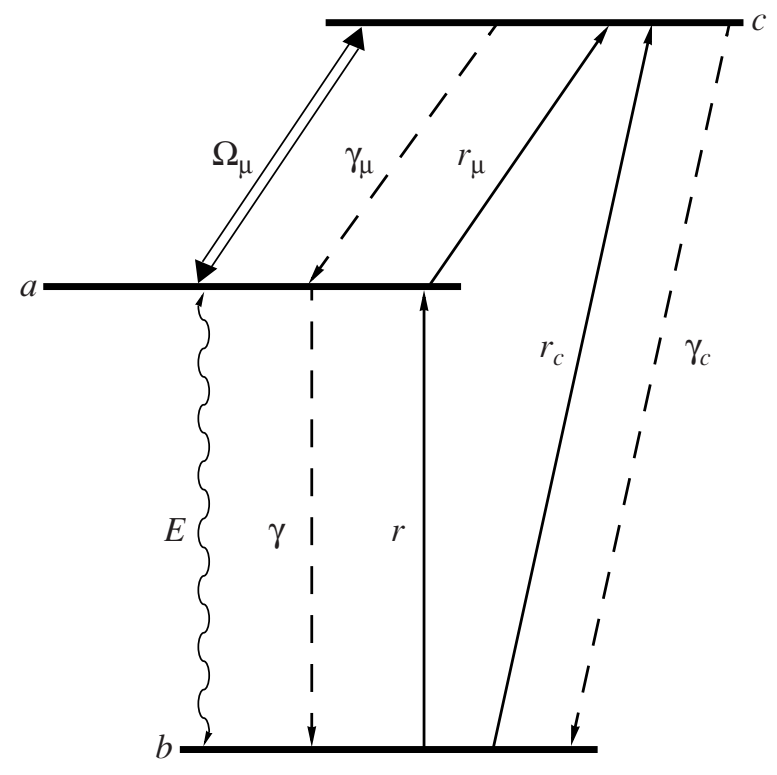

Fig. 1. Upper-level microwave scheme for index enhancement [14]. Upper two levels $a$ and $c$ are coupled via a strong microwave field of Rabi frequency $\Omega_{\mu}$. Weak probe field $E$, of optical frequency $\omega$ is coupled to levels $a$ and $b$. Decay $(\gamma)$ and pump $(r)$ rates are indicated.

nents to the bands of PC [16-22]. In the widely used Drude model, absorption is of negligible importance in the transparency window. Small, but realistic, amount of absorption does not change the band structure much [18]. On the other hand in the region of appreciable absorption there may occur no gap [21].

The dielectric function, we deal here, is completely different then the ones considered up to present. BEC offers gain regimes, as well as absorptive, beyond the enhancement window [14], which resembles the lasing without inversion [15]. To our knowledge, PBG of such a periodic structure, composed of index-enhanced media, is investigated in [13] for the first time. The gain regime is important for the understanding of the lasing properties of PC [23], as well as absorptive properties are advantageous in various applications [24].

The paper is organized as follows. In Section 2 we describe the dielectric function $\epsilon(\mathbf{r}, \omega)$ inside the vortex $\mathrm{PC}$, that is enhanced with upper-level microwave scheme [14]. In Section 3, we deduce the matrix equations from the master equation of PC. We show how to obtain the band structure for a complex frequencydependent dielectric susceptibility. In Section 4.1 we present the resulting photonic bands for two different lattice constants. In Section 4.2 we discuss the frequency width of the band gap in the presence of gain and absorption. In Section 5 we present the band results for Raman index enhancement scheme. We summarize our results in Section 6.
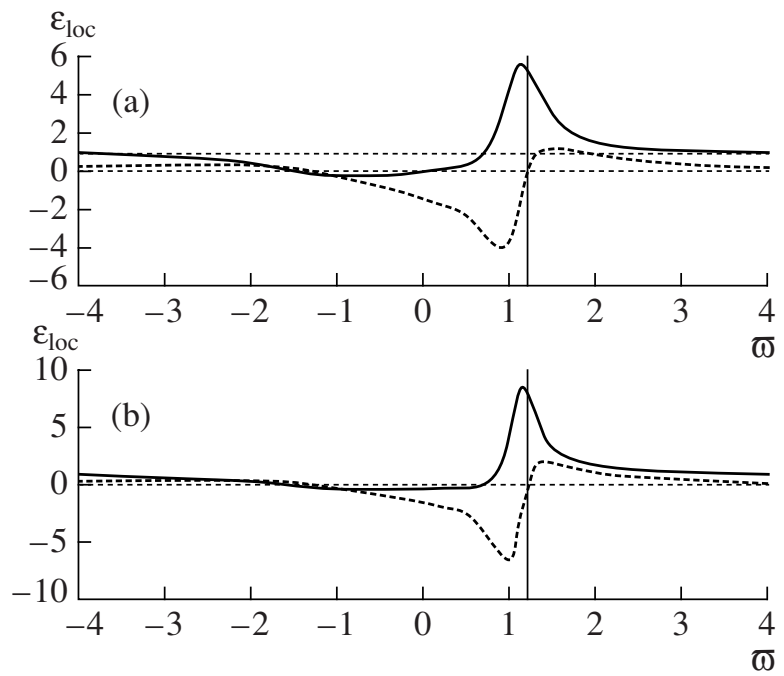

Fig. 2. Real (solid-line) and imaginary (doted-line) parts of local dielectric function $\epsilon_{\mathrm{loc}}(\omega)$ as a function of scaled frequency $\bar{\omega}=\left(\omega-\omega_{a b}\right) / \gamma$, for the particle densities (a) $N=$ $5.5 \times 10^{20}$ and (b) $6.6 \times 10^{20} \mathrm{~m}^{-3}$. Vertical solid line indicates the scaled enhancement frequency $\bar{\varpi}_{0} \simeq 1.22$, where $\epsilon_{\text {loc }}^{\prime \prime}(\varpi)$ vanishes. (a) $\epsilon=\epsilon_{\text {loc }}\left(\varpi_{0}\right)=5.2$ and (b) 8.0.

\section{DIELECTRIC FUNCTION OF THE VORTEX LATTICE}

Among the various index enhancement schemes [14], upper-level microwave scheme (see Fig. 1) leads to the strongest index contrast with vanishing absorption (Fig. 2). Another one is the Raman scheme [14], discussed in Section 5, generates weaker enhancement, but in a broader frequency window. The former one results in stronger stoppage at a single frequency, while the later operates as a weaker blocker at broader frequency range.

In the upper-level microwave scheme, the excited level $a$ is coupled to an auxiliary level $c$ via a strong resonant microwave field of Rabi frequency $\Omega_{\mu}$. The weak optical field $E$, coupling the ground state $b$ to excited state $a$, is the test field. Its dielectric response is calculated. Alternative absorption path $b \longrightarrow c \longrightarrow a$ destructively interfere with the direct absorption path $b \longrightarrow a$ [15]. This quantum interference, due to the phase difference, cancel the absorption of the probe pulse $E$ at a certain frequency, plotted in Fig. 2. At the same time, high polarization is obtained by keeping population at the excited level $a$.

Upper-microwave scheme, for $r_{c}=\Omega_{\mu}=\gamma$, results in the complex dielectric susceptibility $\chi(\omega)=\chi^{\prime}(\omega)+$ $i \chi^{\prime \prime}(\omega)$ of real $\chi^{\prime}$ and imaginary parts $\chi^{\prime \prime}$ are [14]

$$
\chi^{\prime}(\varpi)=\frac{12 N \lambda^{3}}{13 \pi^{2}} \frac{\varpi}{9-3 \bar{\varpi}^{2}+4 \varpi^{4}},
$$

